Advancing Computing as a Science \& Profession

\title{
IUI'15 Companion
}

Companion of the 20th ACM International Conference on Intelligent User Interfaces

Sponsored by:

\section{ACM SIGAI and ACM SIGCHI}

Supported by:

Google, Microsoft Research, Yahoo Labs, eBay, HPCC Systems, and Symantec 
Association for

Computing Machinery

Advancing Computing as a Science \& Profession

\section{The Association for Computing Machinery 2 Penn Plaza, Suite 701 New York, New York 10121-0701}

Copyright (C) 2015 by the Association for Computing Machinery, Inc. (ACM). Permission to make digital or hard copies of portions of this work for personal or classroom use is granted without fee provided that copies are not made or distributed for profit or commercial advantage and that copies bear this notice and the full citation on the first page. Copyright for components of this work owned by others than ACM must be honored. Abstracting with credit is permitted. To copy otherwise, to republish, to post on servers or to redistribute to lists, requires prior specific permission and/or a fee. Request permission to republish from: permissions@acm.org or Fax +1 (212) 869-0481.

For other copying of articles that carry a code at the bottom of the first or last page, copying is permitted provided that the per-copy fee indicated in the code is paid through www.copyright.com.

Notice to Past Authors of ACM-Published Articles

ACM intends to create a complete electronic archive of all articles and/or other material previously published by ACM. If you have written a work that has been previously published by ACM in any journal or conference proceedings prior to 1978, or any SIG Newsletter at any time, and you do NOT want this work to appear in the ACM Digital Library, please inform permissions@acm.org, stating the title of the work, the author(s), and where and when published.

ISBN: 978-1-4503-3308-5

Additional copies may be ordered prepaid from:

\section{ACM Order Department}

PO Box 30777

New York, NY 10087-0777, USA

Phone: 1-800-342-6626 (USA and Canada)

$+1-212-626-0500$ (Global)

Fax: +1-212-944-1318

E-mail: acmhelp@acm.org

Hours of Operation: 8:30 am - 4:30 pm ET

Printed in the USA 


\section{Welcome}

It is with great pleasure that we welcome you to the 2015 ACM International Conference on Intelligent User Interfaces - ACM IUI 2015 in Atlanta, Georgia, USA. Starting in 1993, ACM IUI is now in its 20th edition and serves as a premier international forum for reporting outstanding research and development on intelligent user interfaces. ACM IUI is where the Human-Computer Interaction (HCI) community meets the Artificial Intelligence (AI) community. We are also very interested in contributions from related fields, such as psychology, behavioral science, cognitive science, computer graphics, design, the arts, etc.

At ACM IUI, we focus on the interaction between machine intelligence and human intelligence. While other conferences focus on one side or the other, we address the complex interaction between the two. We welcome research that explores how to make the interaction between computers and people smarter, which may leverage solutions from data mining, knowledge representation, novel interaction paradigms, and emerging technologies.

For the 20th edition of the conference, the call for papers attracted a record number of 205 long and short paper submissions, 41 poster paper submissions, 18 demo paper submissions, and 24 student consortium submissions. The final program of the conference includes 3 keynotes, 47 long and short papers (22.9\% acceptance rate), 17 posters, 8 demos, 4 workshops, 3 tutorials, and 12 student consortium papers. The program opens with a keynote by Professor Dan Weld on "Intelligent Control of Crowdsourcing", continues with a keynote by Ed Chi on "Blurring of the Boundary Between Interactive Search and Recommendation" on the second day, and ends with a keynote by Rosalind Picard on "Recognizing Stress, Engagement, and Positive Emotion". Four workshops (Personalized Access to Cultural Heritage, Intelligent Digital Games for Empowerment and Inclusion, Interacting with Smart Objects, and Visual Text Analytics) and three tutorials (Speech-based Interaction, Personalization for Behavior Change, and User Affect and Sentiment Modeling) complement the main conference program.

The conference could not be organized without the help of a large number of individuals who volunteered a lot of their own time and enthusiasm. Their names can be found on the following pages. The organization committee has worked very hard and a big thank you goes to all people who helped overcome all sorts of problems. We must also thank our senior program committee for coordinating the review process and all the members of the program committee for providing high quality reviews. And, most important, we must thank the authors for providing the content for a never seen number of submissions, and also those whose papers could not be included in the $22.9 \%$ selected for the program. Finally, we must thank our sponsors, ACM, SIGCHI and SIGART, as well as our generous corporate supporters (found on the following pages).

We hope you will find the program interesting and thought-provoking. We also hope the conference will provide you with a valuable opportunity to share ideas with other researchers and practitioners from around the world, leading to further advancements in the field of intelligent user interfaces.

\section{Oliver Brdiczka, Polo Chau Giuseppe Carenini, Shimei Pan, Per Ola Kristensson ACM IUI 2015 General Co-Chairs ACM IUI 2015 Program Co-Chairs}




\section{Table of Contents}

\section{Poster \& Demo Session}

- Automatic Generation and Insertion of Assessment Items in Online Video Courses 1 Amrith Krishna, Plaban Kumar Bhowmick, Krishnendu Ghosh, Archana Sahu, Subhayan Roy (Indian Institute of Technology Kharagpur)

- The News Context Project. Larry Birnbaum, Miriam Boon, Scott Bradley, Jennifer Wilson (Northwestern University)

- MuLS: An Open Source EEG Acquisition and Streaming Server for Quick and Simple Prototyping and Recording Raymundo Cassani, Hubert Banville, Tiago H. Falk (University of Quebec)

- Towards Integrating Real-Time Crowd Advice with Reinforcement Learning 17 Gabriel V. de la Cruz Jr. , Bei Peng (Washington State University), Walter S. Lasecki (University of Rochester), Matthew E. Taylor (Washington State University)

- User-Interfaces for Incremental Recipient and Response Time Predictions in Asynchronous Messaging Connor Hamlet, Daniel Korn, Nikhil Prasad, Volodymyr Siedlecki, Eliezer Encarnacion, Jacob Bartel, Prasun Dewan (University of North Carolina at Chapel Hill)

- Interactive Control and Visualization of Difficulty Inferences from User-Interface Commands

Duri Long, Nicholas Dillon, Kun Wang, Jason Carter, Prasun Dewan (University of North Carolina at Chapel Hill)

- OfficeHours: A System for Student Supervisor Matching through Reinforcement Learning Yuan Gao, Kalle Ilves, Dorota Glowacka (University of Helsinki)

- From Overview to "Detail": An Exploration of Contextual Transparency for Public Transparent Interfaces

Heesun Kim, BoKyung Huh, SeungHyen Im, HaeYoun Jung, Gyu Hyun Kwon, Ji-Hyung Park

(Korea Institute of Science and Technology)

- WallSHOP: Multiuser Interaction with Public Digital Signage using Mobile Devices for Personalized Shopping

Soh Masuko, Masafumi Muta, Keiji Shinzato, Adiyan Mujibiya (Rakuten, Inc.)

- Surveying Older Adults About a Recommender System for a Digital Library. Adam N. Maus, Amy K. Atwood (University of Wisconsin - Madison)

- A Task-Centered Interface for On-Line Collaboration in Science.

Felix Michel, Yolanda Gil, Varun Ratnakar (University of Southern California), Matheus Hauder (Technical University Munich)

- VizRec:A Two-Stage Recommender System for Personalized Visualizations Belgin Mutlu, Eduardo Veas, Christoph Trattner, Vedran Sabol (Know Center)

- Mechanix: A Sketch-Based Educational Interface Trevor Nelligan, Seth Polsley, Jaideep Ray (Texas A\&M University), Michael Helms, Julie Linsey (Georgia Institute of Technology), Tracy Hammond (Texas A\&M University)

- An Interactive Pedestrian Environment Simulator for Cognitive Monitoring and Evaluation Jason Orlosky, Markus Weber, Yecheng Gu, Daniel Sonntag, Sergey Sosnovsky (German Research Center for Artificial Intelligence)

- Interactive Querying over Large Network Data: Scalability, Visualization, and Interaction Design 
Robert Pienta, Acar Tamersoy (Georgia Institute of Technology), Hanghang Tong (Arizona State University), Alex Endert, Duen Horng Chau (Georgia Institute of Technology)

- Robot Companions and Smartpens for Improved Social Communication of Dementia Patients.

Alexander Prange, Indra Praveen Sandrala, Markus Weber, Daniel Sonntag (German Research Center for AI)

- Data Privacy and Security Considerations for Personal Assistants

for Learning (PAL)

Elaine M. Raybourn (Sandia National Labs \& Advanced Distributed Learning Initiative),

Nathan Fabian, Warren L. Davis IV, Ray Parks, Jonathan T. McClain, Derek Trumbo (Sandia National Labs),

Damon Regan, Paula J. Durlach (Advanced Distributed Learning Initiative)

- Framework for Realizing a Free-Target Eye-tracking System

Daiki Sakai, Michiya Yamamoto (Kwansei Gakuin University),

Takashi Nagamatsu (Technical University of Munich \& Kobe University)

- Intelligent Search for Biologically Inspired Design

Evangelia Spiliopoulou, Spencer Rugaber, Ashok Goel, Lianghao Chen, Bryan Wiltgen,

Arvind Krishnaa Jagannathan (Georgia Institute of Technology)

- USHER: An Intelligent Tour Companion

Shubham Toshniwal, Parikshit Sharma, Saurabh Srivastava (IBM Research), Richa Sehgal (IBM SWG)

- Human-Machine Cooperative Viewing System for Wide-angle Multi-view Videos .85

Fumiharu Tomiyasu, Kenji Mase (Nagoya University)

- Hairware: Conductive Hair Extensions as a Capacitive Touch Input Device Katia Vega, Marcio Cunha, Hugo Fuks (Pontifical Catholic University of Rio de Janeiro)

- Mindminer: Quantifying Entity Similarity via Interactive Distance Metric Learning Xiangmin Fan, Youming Liu (University of Pittsburgh), Nan Cao (IBM T.J. Watson Research Center), Jason Hong (Carnegie Mellon University), Jingtao Wang (University of Pittsburgh)

- A Model for Data-Driven Sonification Using Soundscapes.

KatieAnna E. Wolf, Genna Gliner (Princeton University), Rebecca Fiebrink (University of London)

\section{Student Consortium}

- Towards a Crowd-based Picture Schematization System

Huaming Rao (Nanjing University of Science and Technology)

- Visual Text Analytics for Asynchronous Online Conversations.

Enamul Hoque (University of British Columbia)

- Real-Time Emotion Detection for Neuro-Adaptive Systems Kathrin Pollmann (University of Stuttgart)

- Clinical Text Analysis Using Interactive Natural Language Processing

Gaurav Trivedi (University of Pittsburgh)

- Perceptive Home Energy Interfaces:

Navigating the Dynamic Household Structure

Germaine Irwin (University of Maryland, Baltimore County)

- On-Body Interaction for Optimized Accessibility ... David Costa (Universidade de Lisboa)

- Assisting End Users in the Design of Sonification Systems KatieAnna E. Wolf (Princeton University)

- Multimodal Interactive Machine Learning for User Understanding Xuan Guo (Rochester Institute of Technology)

- A Revisit to the Identification of Contexts in Recommender Systems 133 Yong Zheng (DePaul University)

- AmbLEDs: Context-Aware I/O for AAL Systems Marcio Cunha (Pontifical Catholic University of Rio de Janeiro) 
- Know Your Surroundings with an Interactive Map

Sanorita Dey (University of Illinois at Urbana-Champaign)

- Extended Virtual Presence of Therapists Through Home Service Robots.

Hee-Tae Jung (University of Massachusetts, Amherst)

Author Index. 


\section{ACM IUI 2015 International Conference on Intelligent User Interfaces - Organization}

General Chairs: Oliver Brdiczka (Vectra Networks, Inc.)

Polo Chau (Georgia Tech)

Program Chairs: Giuseppe Carenini (University of British Columbia)

Shimei Pan (University of Maryland)

Per Ola Kristensson (University of Cambridge)

Treasurer: Jalal Mahmud (IBM Research)

Sponsorship Chair: Sumit Basu (Microsoft Research)

Workshop and Tutorial Ben Steichen (University of British Columbia)

Chairs: Nava Tintarev (University of Aberdeen)

Poster and Demo Chairs: Sven Kratz (FXPAL)

Eelco Herder (L3S)

Student Consortium Wai-Tat Fu (University of Illinois at Urbana-Champaign)

Chairs: Brent Hecht (University of Minnesota)

Publicity Chairs: Matthijs van Leeuwen (KU Leuven)

Jilles Vreeken (Max Planck Institute of Informatics)

Alan Said (Recorded Future)

Ali Jahanian (Purdue University)

\section{Senior Program Committee:}

Gregory Abowd

Elisabeth Andre

Liliana Ardissono

Sumit Basu

Shlomo Berkovsky

Pradipta Biswas

Fang Chen

Ashok Goel

Program Committee:

Safurah Abdul Jalil

Fadel Adib

Aaron Adler

Ana Afonso

Elena Agapie
Joaquim Jorge

Matthijs van Leeuwen

Henry Lieberman

Christian Mueller

Petteri Nurmi

Nuria Oliver

Cecile Paris

Fabio Paterno

Imtiaj Ahmed

Dzmitry Aliakseyeu

Bilal Alsallakh

Florian Alt

Saleema Amershi
Catherine Pelachaud

Chris Schmandt

Amanda Stent

Simone Stumpf

Manas Tungare

Keith Vertanen

Jilles Vreeken

Chen $\mathrm{Yu}$
Anima Anandkumar

Fraser Anderson

Salvatore Andolina

Michelle Annett

Craig Anslow 


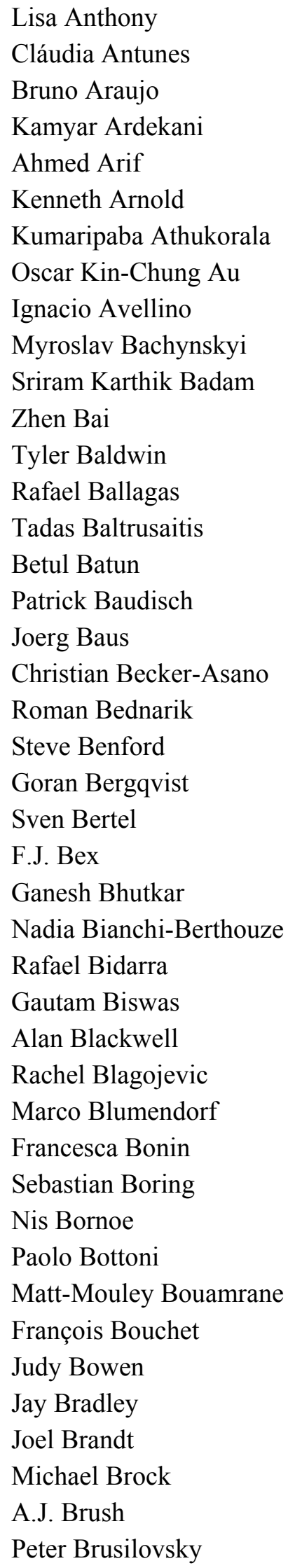

\begin{tabular}{|c|c|}
\hline George Buchanan & Franco Curmi \\
\hline Andreas Bulling & Aba-Sah Dadzie \\
\hline Andrea Bunt & Florian Daiber \\
\hline Margaret Burnett & Ionut Damian \\
\hline Jim Burton & Andreea Danielescu \\
\hline Heloisa Candello & Sauvik Das \\
\hline Angelo Cafaro & Scott Davidoff \\
\hline Pável Calado & Nadja De Carolis \\
\hline Gaelle Calvary & Luigi De Russis \\
\hline Pedro Campos & Aidan Delaney \\
\hline Jose Campos & Nicola Dell \\
\hline Karina Caro & Alexandre Demeure \\
\hline Juan Pablo Carrascal & Carrie Demmans Epp \\
\hline Luis Carriço & Sarah Diefenbach \\
\hline Mark Cartwright & Stefan Diewald \\
\hline Jessica Cauchard & Shalaka Dighe \\
\hline Marc Cavazza & Karthik Dinakar \\
\hline Federica Cena & Anke Dittmar \\
\hline Daniel Cernea & Divya Bansal Divya Bansa \\
\hline Pablo Cesar & Paul Dourish \\
\hline Allison Chaney & Fan $\mathrm{Du}$ \\
\hline Samir Chatterjee & Sumit Gulwani \\
\hline Debaleena Chattopadhyay & Carlos Duarte \\
\hline Ge Chen & Andrew Duchowski \\
\hline Xiang 'Anthony' Chen & Henry Duh \\
\hline Zhenyu Chen & Cody Dunne \\
\hline Alex Qiang Chen & Varun Dutt \\
\hline Jingyuan Cheng & James Eagan \\
\hline Keith Cheverst & David England \\
\hline Jun Dong Cho & Barrett Ens \\
\hline Mathieu Chollet & Irfan Essa \\
\hline Ming Ki Chong & Bernd Ewert \\
\hline Jason Chuang & Myriam Frejus \\
\hline Dahee Chung & Robert Farrell \\
\hline Karen Church & Andrew Faulring \\
\hline José Coelho & Karla Felix Navarro \\
\hline Nathalie Colineau & Tom Feltwell \\
\hline Ashley Colley & Jennifer Ferreira \\
\hline Kay Connelly & Denzil Ferreira \\
\hline Amelie Cordier & Francesco Ferrise \\
\hline Mark Cottman-Fields & Bruce Ferwerda \\
\hline Michael Crabb & Rebecca Fiebrink \\
\hline Sally Jo Cunningham & Leah Findlater \\
\hline
\end{tabular}

Franco Curmi

Ionut Damian

adja De Carolis

icola Dell

Sarah Diefenbach

Shalaka Dighe

Anke Dittmar

Divya Bansal Divya Bansal

Fan Du

Sumit Gulwani

Andrew Duchowski

Cody

Cody Dunne

James Eagan
Scott Davidoff 


Daniel Fitton
David Flatla
Scott Fleming
Eelke Folmer
Manuel Fonseca
Angus Forbes
Andrew Fowler
Jill Freyne
Christopher Fry
Chi-Wing Fu
Jochen Huber
Steve Funke
Susanne Furman
Krzysztof Gajos
William Gaver
Patrick Gebhard
Sven Gehring
Gretchen Gelke
Hans Gellersen
Cristina Gena
Werner Geyer
Giuseppe Ghiani
Marco Gillies
Romain Giot
Patrick Girard
Elena Glassman
Mayank Goel
Jennifer Golbeck
Steven Gomez
David Gotz
Liang Gou
Visda Goudarzi
Sten Govaerts
Anna Goy
Nitesh Goyal
Saul Greenberg
Tobias Grosse-Puppendahl
Paul Groth
Jens Grubert
Ean Gruen

\begin{tabular}{|c|c|}
\hline Philip Guo & Sampath Jayarathna \\
\hline Rongkai Guo & Haiyan Jia \\
\hline Anhong Guo & Jiepu Jiang \\
\hline Sidhant Gupta & Priscilla Jimenez Pazmino \\
\hline Eben Haber & Bonnie John \\
\hline Jonna Hakkila & Michael Johnston \\
\hline Scott Hale & Brian Jones \\
\hline Martin Halvey & Park Joongsin \\
\hline William Hamilton & Tejinder Judge \\
\hline Tracy Hammond & Hyunggu Jung \\
\hline Kotaro Hara & Marko Jurmu \\
\hline Marian Harbach & Antti Jylhä \\
\hline Erik Harpstead & Pradyot $\mathrm{K}$ biswas \\
\hline Daniel Harrison & Gerrit Kahl \\
\hline Chris Harrison & Sanjay Kairam \\
\hline Kirstie Hawkey & Santosh Kalwar \\
\hline Brent Hecht & Alexandros Karatzoglou \\
\hline Elliott Hedman & Sarvnaz Karimi \\
\hline Christin Henzen & Kostas Karpouzis \\
\hline Eelco Herder & Pedro Lopes \\
\hline Thomas Hermann & Pasquale Lops \\
\hline Ken Hinckley & Konstantinos Kazakos \\
\hline Liangjie Hong & Rubaiat Habib Kazi \\
\hline Sungsoo (Ray) Hong & Simeon Keates \\
\hline Enamul Hoque & Kerstin Keil \\
\hline Eva Hornecker & Caitlin Kelleher \\
\hline Steven Houben & Maryam Khademi \\
\hline Pei-Yun Hsueh & Mohammadreza Khalilbeigi \\
\hline Mengdie $\mathrm{Hu}$ & Peter Kiefer \\
\hline Mao Huang & Jeeeun Kim \\
\hline Andrew Kun & HyungSeok Kim \\
\hline Pei-Yi Kuo & Reuben Kirkham \\
\hline Shah Rukh Humayoun & Felix Kistler \\
\hline Axel Hösl & Styliani Kleanthous \\
\hline Kori Inkpen & Mark Klein \\
\hline Stephen Intille & Franziska Klüg1 \\
\hline Shamsi Iqbal & Peter Knees \\
\hline Tobias Isenberg & Søren Knudsen \\
\hline Jason Jacques & Andrew Ko \\
\hline Javier Jaen & Christian Koehler \\
\hline Mohit Jain & Boriana Koleva \\
\hline Dietmar Jannach & Oleg Komogortsev \\
\hline Caroline Jay & Matthias Kranz \\
\hline
\end{tabular}


Kyriakos Kritikos

Jan-Peter Krämer

Antonio Krüger

Thomas Kubitza

Tsvi Kuflik

Todd Kulesza

Arun Kulshreshth

Vikas Kumar

Alex Mitchell

Monika Mitrevska

Darya Kurilova

Sandeep Kaur Kuttal

Bum Chul Kwon

Andol LI

Thomas LaToza

Shyong Lam

Patrick Langdon

Walter Lasecki

Neal Lathia

Yann Laurillau

Sungjin Lee

Uichin Lee

Chaiwoo Lee

Jong-Seok Lee

Luis A. Leiva

Catherine Letondal

Margot Lhommet

Jane Li

Shuowei Li

Thomas Lieber

Henry A. Lieberman

James Lin

Jimmy Lin

Rhema Linder

Lars Lischke

Meizhu Liu

Ying Hsang Liu

Tomek Loboda

Ronan Longergan

Ricardo Lopes

Zhihan Lv

Alex Olwal

Tihomir Orehovacki
Pilar Orero

Zhihan Lu

Jie Lu

Bernd Ludwig

Christopher Lueg

Kurt Luther

Kris Luyten

Markus Löchtefeld

Lindsay MacDonald

Blair MacIntyre

Allan MacLean

Haley MacLeod

Sylvain Malacria

Marco Manca

Jennifer Mankoff

Jennifer Marlow

Diogo Marques

William Marsh

Roberto Martinez Maldonado

Mikhil Masli

Cindy Mason

Masood Masoodian

Tara Matthews

Michael Mauderer

Christopher Mayer

David McGee

Daniel Mendes

Xiaojun Meng

Rafał Michalski

Robert Miller

Fatemeh Rajabiyazdi

Ramesh Raju

Raf Ramakers

Manas Mittal

Leonel Morales Diaz

Fabio Morreale

Ahmed E. Mostafa

Christos Mousas

Adiyan Mujibiya

Cosmin Munteanu

Emerson Murphy-Hill

Mirco Musolesi

Bilge Mutlu
Frank Nack

Hideyuki Nakanishi

Yukiko Nakano

Kim Nam Wook

N. Hari Narayanan

Fedelucio Narducci

Majid Nasirinejad

Michael Nebeling

Surya Nepal

Grace Ngai

Tien Nguyen

Karin Niemantsverdriet

Onyinyechukwu Nnadi

(chigbo)

Pedro Nogueira

Diana Nowacka

Nuno Nunes

John O'Donovan

Timo Ojala

Thomas Olsson

Arvind Satyanarayan

Christopher Scaffidi

Florian Schaub

Jason Orlosky

Lucas Paletta

Ye Pan

Aasish Pappu

Alexandros Paramythis

Deokgun Park

Souneil Park

Aman Parnami

Paul Parsons

Evan Peck

Ruggero Pensa

Lucas Pereira

Giovanna Petrone

Tamara Peyton

Martin Pielot

Thomas Pietrzak

Laura Pina

Till Plumbaum

Scott Pobiner

Benjamin Poppinga 


Christopher Power
Rui Prada
María Prato
Felix Putze
Yin Qu
Pernilla Qvarfordt
Frederic Raber
Emilee Rader
Erin Solovey
Yale Song
Daniel Sonntag
Danny Soroker
Martin Raubal
Khasfariyati Razikin
Janet Read
Sofia Reis
Steven Reiss
Norbert Reithinger
Yosra Rekik
Christian Remy
Kui Ren
Philippe Renevier Gonin
Peter Robinson
Simon Robinson
Michael Rohs
Elizabeth Rosenzweig
Thijs Roumen
Rebekah Rousi
Paulami Roy Choudhury
Spencer Rugaber
Nicolas Sabouret
Ramik Sadana
Alireza Sahami Shirazi
Alan Said
André Salgado
Jose San Pedro
Carmen Santoro
Olga Santos
Sanat Sarangi

\begin{tabular}{|c|c|}
\hline Huawei Tu & Yuta Sugiura \\
\hline Michael Schellenbach & Bongwon Suh \\
\hline Stefan Scherer & Shamik Sural \\
\hline Gianluca Schiavo & Craig Sutherland \\
\hline Albrecht Schmidt & Federico Tajariol \\
\hline Dominik Schmidt & Peter Thomas \\
\hline Martin Schmitz & Edison Thomaz \\
\hline Oliver Schneider & Feng Tian \\
\hline Stacey Scott & Nava Tintarev \\
\hline Kenneth Scott-Brown & Marko Tkalcic \\
\hline Michael Sedlmair & Kashyap Todi \\
\hline Hasti Seifi & Kashyap Todi \\
\hline Ted Selker & Dereck Toker \\
\hline Jonghoon Seo & Mercan Topkara \\
\hline Jinwook Seo & Thomas Torsney-weir \\
\hline Teddy Seyed & Takumi Toyama \\
\hline Metin Sezgin & Scott Trent \\
\hline Orit Shaer & Vlad Trifa \\
\hline S. Andrew Sheppard & Victor de Boer \\
\hline Roy Shilkrot & Lilian de Greef \\
\hline Frank Shipman & Alwin de Rooij \\
\hline Sajad Shirali-Shahreza & Anuradha Uduwage \\
\hline Mark Shovman & Heli Väätäjä \\
\hline Paula Alexandra Silva & Haley Vaigneur \\
\hline Aneesha Singh & Dimitar Valkov \\
\hline Pushpendra Singh & Max Van Kleek \\
\hline R. Singh & Herwin van Welbergen \\
\hline Laurianne Sitbon & Jean Vander Donckt \\
\hline Jan Smeddinck & Keith Vander Linden \\
\hline Mathias Wilhelm & Radu-Daniel Vatavu \\
\hline Jason D Williams & Swaroop Vattam \\
\hline John Williamson & Ansh Verma \\
\hline Maria Wolters & Jo Vermeulen \\
\hline Caleb Southern & Emanuel von Zezschwitz \\
\hline Lucio Davide Spano & Romain Vuillemot \\
\hline Lucio Davide Spano & Erin Walker \\
\hline Lübomira Spassova & James Wallace \\
\hline Rob Speer & Wen Wang \\
\hline Robert St. Amant & Tao Wang \\
\hline Richard Stallman & Na Wang \\
\hline Chad Steed & Ji Wang \\
\hline Ben Steichen & Jingtao Wang \\
\hline Svetlana Stoyanchev & William Yang Wang \\
\hline
\end{tabular}


Morten Warncke-Wang

Gloria Washington

Rainer Wasinger

Daryl Weir

Justin Weisz

Komminist Weldemariam

Weng-Keen Wong

Kanit Wongsuphasawat

Liming Xu

Anbang Xu

Qianli Xu

Poster \& Demo Reviewers:

Ragaad Al Tarawneh

Michelle Annett

Liliana Ardissono

Bo Begole

Shlomo Berkovsky

Federico Botella

Gaelle Calvary

Michael Crabb

Luigi De Russis

Vania Dimitrova

Fan Du

Carlos Duarte

Guihuan Feng

Bruce Ferwerda

Chris Frisina

Steve Funke

Leman Gul

Tracy Hammond

Michael Heidt

Christin Henzen

Eelco Herder

Shah Rukh Humayoun

Germaine Irwin

Dhiraj Joshi

Student Consortium Mentors:

Elizabeth Andre

Joyce Chai

Krzysztof Gajos
Yi Yang

Svetlana Yarosh

Yina Ye

Sang Ho Yoon

Neil Yorke-Smith

James Young

Kun $\mathrm{Yu}$

Chen-Hsiang $\mathrm{Yu}$

John Zaientz

Massimo Zancanaro

Cheng Zhang

Jeeeun Kim

Styliani Kleanthous

Bart Knijnenburg

Soren Knudsen

Birgitta Koenig-Ries

Sven Kratz

Tsvi Kuflik

Rodrigo Laiola Guimaraes

Shyong Lam

Samantha LeVan

Agnes Lisowska Masson

Ying Hsang Liu

Chulhong Min

Toni-Jan Keith Monserrat

Frank Nack

Petteri Nurmi

Jason Orlosky

Sameer Patil

Celeste Paul

Pernilla Qvarfordt

Fatemeh Rajabiyazdi

Raf Ramakers

Christian Remy

Philippe Renevier Gonin
Bo Zhang

Kunpeng Zhang

Tong Zhang

Dejin Zhao

Yu Zhong

Jianlong Zhou

Kening Zhu

Roshanak Zilouchian-

Moghaddam

John Zimmerman

Arkaitz Zubiaga

Michael Rohs

Thijs Roumen

Alan Said

Yann Savoye

Ben Steichen

Carlo Strapparava

Simone Stumpf

$\mathrm{Wu}-\mathrm{Chen} \mathrm{Su}$

Zachary Toups

Christoph Trattner

Nikos Tsourakis

Ilyas Uyanik

Emanuel von Zezschwitz

Romain Vuillemot

Yulin Wang

Pawel Wozniak

Huichuan Xia

Guo Yu

Xiaojun Yuan

Lv Zhihan

Kening Zhu

Arkaitz Zubiaga

Robert St. Amant

Michelle Zhou 


\section{ACM IUI 2015 Sponsors \& Supporters}
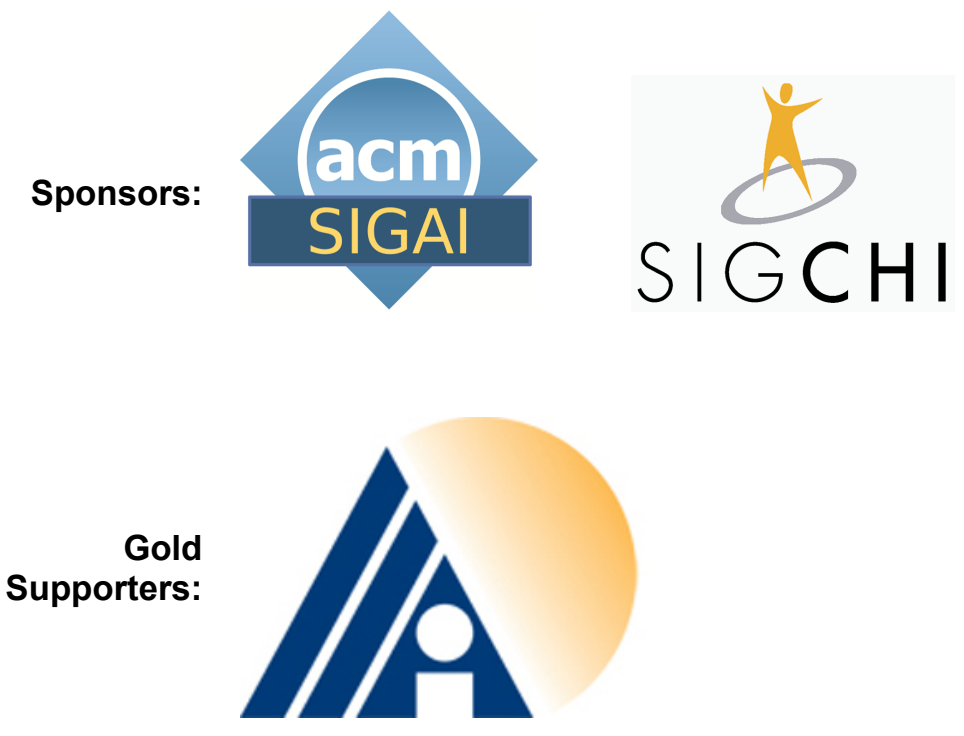

suposteres: Google "Miciosoft

Bronze Supporters:
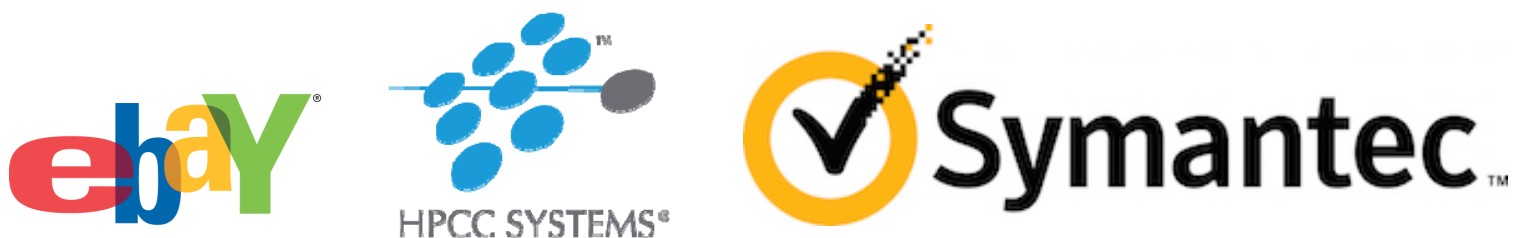

Lanyard:

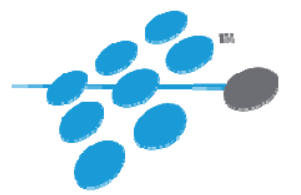

HPCC SYSTEMS ${ }^{\circ}$ 